\title{
Transmission of MRSA CC398 strains between pig farms related by trade of animals
}

Gongora, Carmen Espinosa; Broens, E.M.; Moodley, Arshnee; Nielsen, Jens Peter; Guardabassi, Luca

Publication date:

2011

Document version

Early version, also known as pre-print

Citation for published version (APA):

Gongora, C. E., Broens, E. M., Moodley, A., Nielsen, J. P., \& Guardabassi, L. (2011). Transmission of MRSA CC398 strains between pig farms related by trade of animals. Poster session presented at ASM-ESCMID Conference on Methicillin-resistant Staphylococci in Animals, Washington DC, United States. 


\section{Transmission of MRSA CC398 strains between pig farms related by trade of animals}

\section{Espinosa-Gongora ${ }^{1}$, E. M. Broens ${ }^{2}$, 3 , A. Moodley ${ }^{1}$, J. P. Nielsen ${ }^{4}$, L. Guardabassi ${ }^{1}$.}

${ }^{1}$ Department of Veterinary Disease Biology, Faculty of Life Sciences, University of Copenhagen, Frederiksberg C, Denmark. ${ }^{2}$ Quantitative Veterinary Epidemiology Group, Wageningen Institute of Animal Sciences, Wageningen University, The Netherlands ${ }^{3}$ Centre for Infectious Disease Control Netherlands, National Institute for Public Health and the Environment, Bilthoven, The Netherlands ${ }^{4}$ Department of Large Animal Sciences, Faculty of Life Sciences, University of Copenhagen, Frederiksberg C, Denmark.

Background: Purchasing pigs from a positive farm may be an important risk factor for acquisition of MRSA CC398 in the recipient farms (Broens et al., 2010).

Objective: to provide conclusive molecular evidence that MRSA CC398 is transmitted vertically through the pig production chain by trade of MRSA-positive animals. Our hypothesis was that PFGE profiles found in the recipient farms would also be present in the corresponding supplying farms.

Methods: Six chains (group of farms related to each other by trade of animals) were included in the retrospective study (Chains F, G, P, I, Q, R). Two farms were included the prospective study (Farm 1 and Farm 2) where newly purchased gilts were sampled before entering the farm. Pools of samples from inside the farms and samples from these farms from 2009 were also included (Farm isolates). MRSA isolates from farms linked by commercial trade of pigs were typed using the PFGE protocol by Bosch et al., 2010, a highly discriminatory method allowing differentiation between different lineages of MRSA CC398 (van Wamel et al., 2010). GelCompar II software was used for the cluster analysis and spa types were assigned following the protocol by Harmsen et al., 2003. The study was structured in two parts: a retrospective study (isolates from the study by Broens et al., 2010) and a prospective study (isolates from Denmark).

Results: The results of the retrospective study and the prospective study are presented in Figures 1 and 2, respectively. In most but not all cases, MRSA transmission by trade was supported by PFGE analysis.

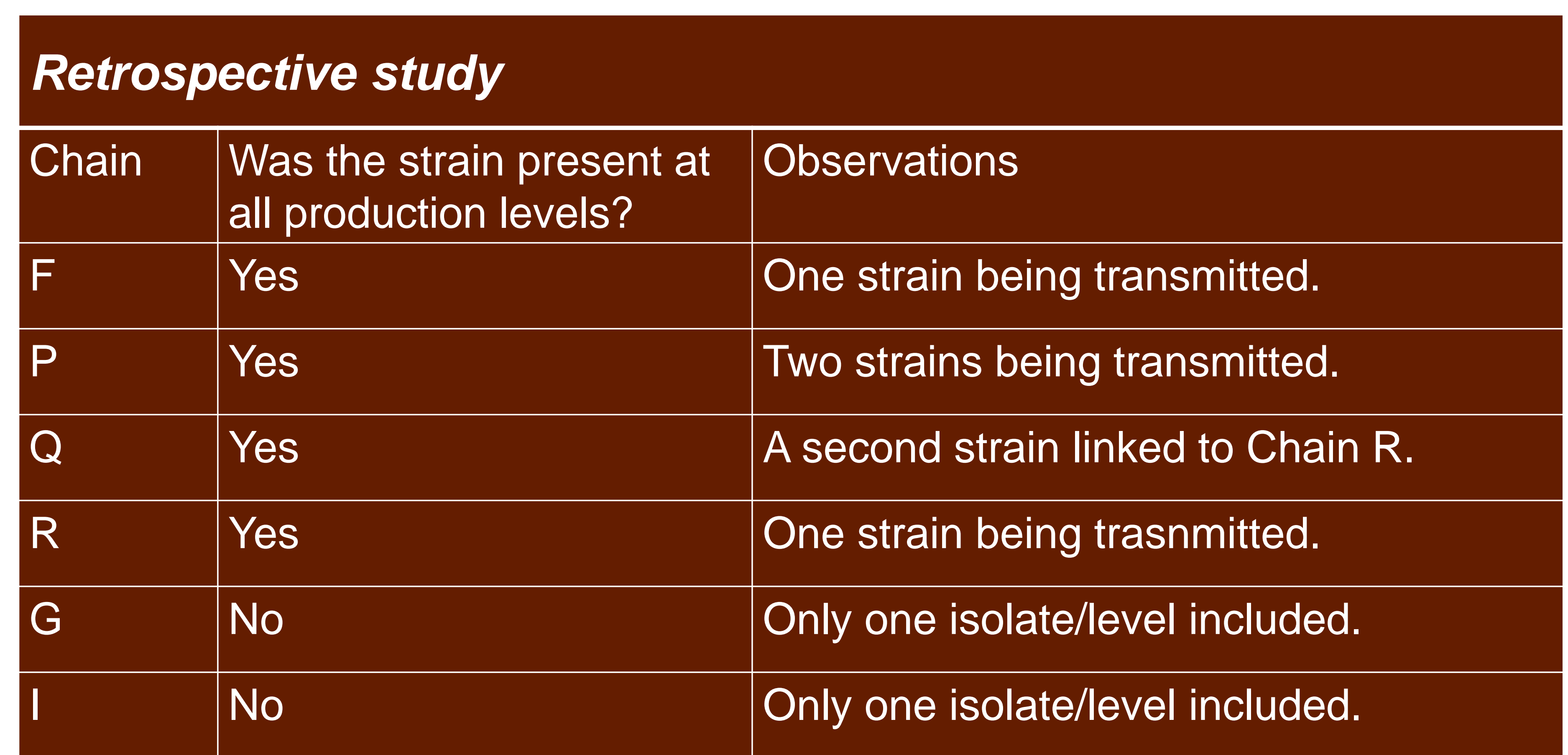

Dice $(0 \mathrm{pt} \mathrm{0} .10 \%)(\mathrm{Tol} 1.0 \%-1.0 \%)(\mathrm{H}>0.0 \% \mathrm{~S}>0.0 \%)[0.0 \%-100 \%$
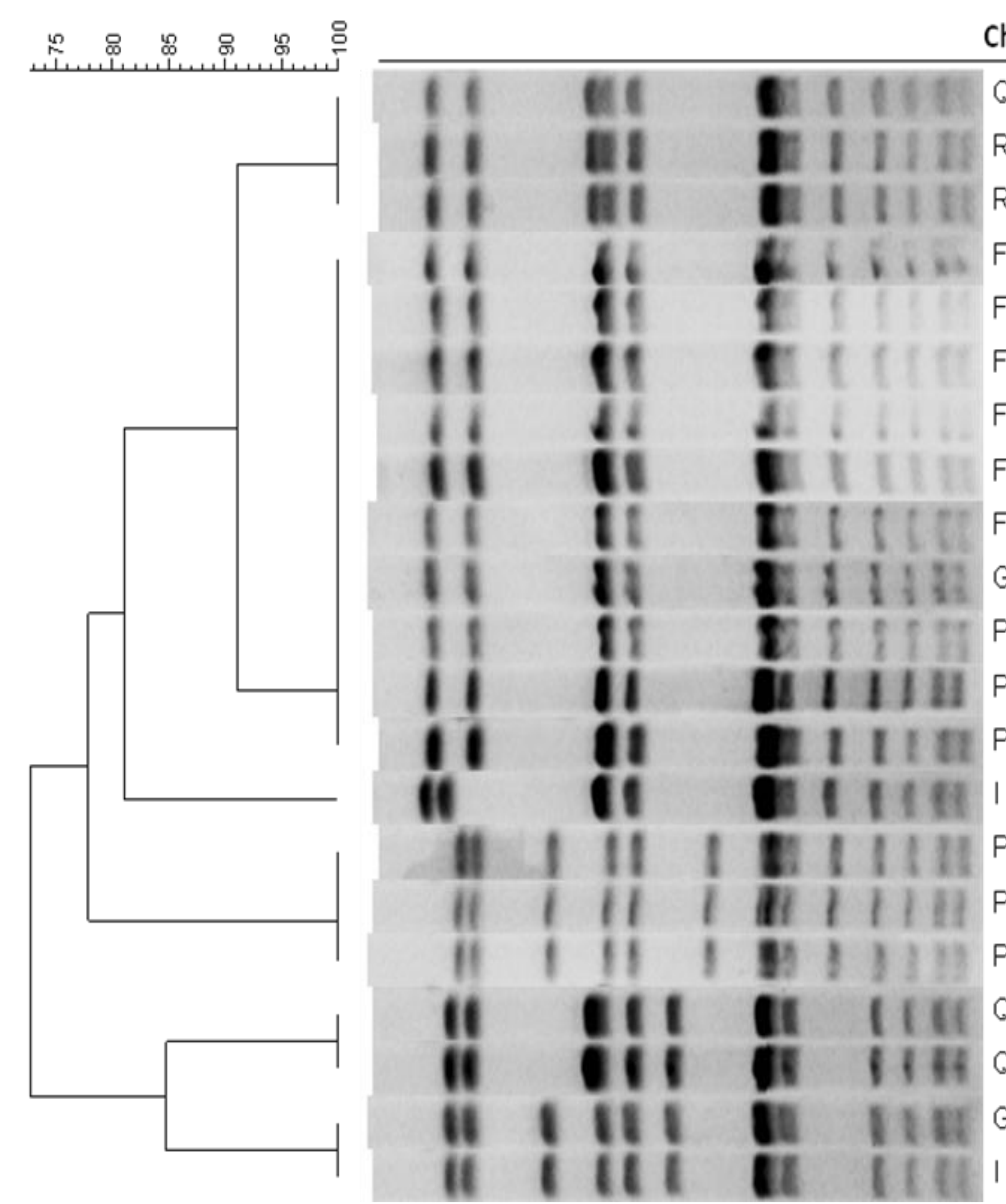

Chain Farm type spatype Farm ID

Figure 1. Genetic diversity of 21 MRSA CC398 isolates from 6 pig farms and their corresponding gilts or pigs suppliers in Holland. Production Chain (F, G, I, P, Q, R), spa type and Farm identification (ID) (1-14) and are provided for each strain.

\section{CONCLUSIONS}

1. MRSA CC398 strains can be transmitted through pig trading, which is important for the development of intervention strategies (Chains F, P, Q, R and Farm 2).

2. Alternative transmission pathways may exist (there are identical strains among farms not related by trade, for example Chains $Q$ and $\mathbf{R})$.

3. Association between PFGE profiles and spa types was not consistent (Figure 1).

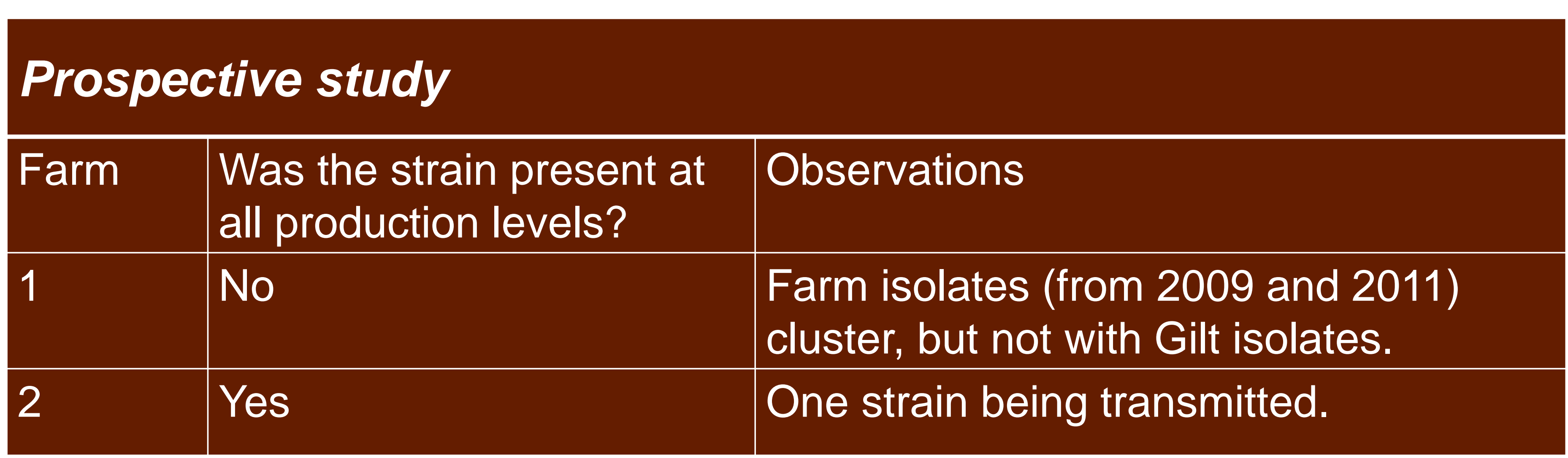

Dice (Opt $0.10 \%)$ (Tol $1.0 \%-1.0 \%)(\mathrm{H}>0.0 \% \mathrm{~S}>0.0 \%)[0.0 \%-100.0 \%)$

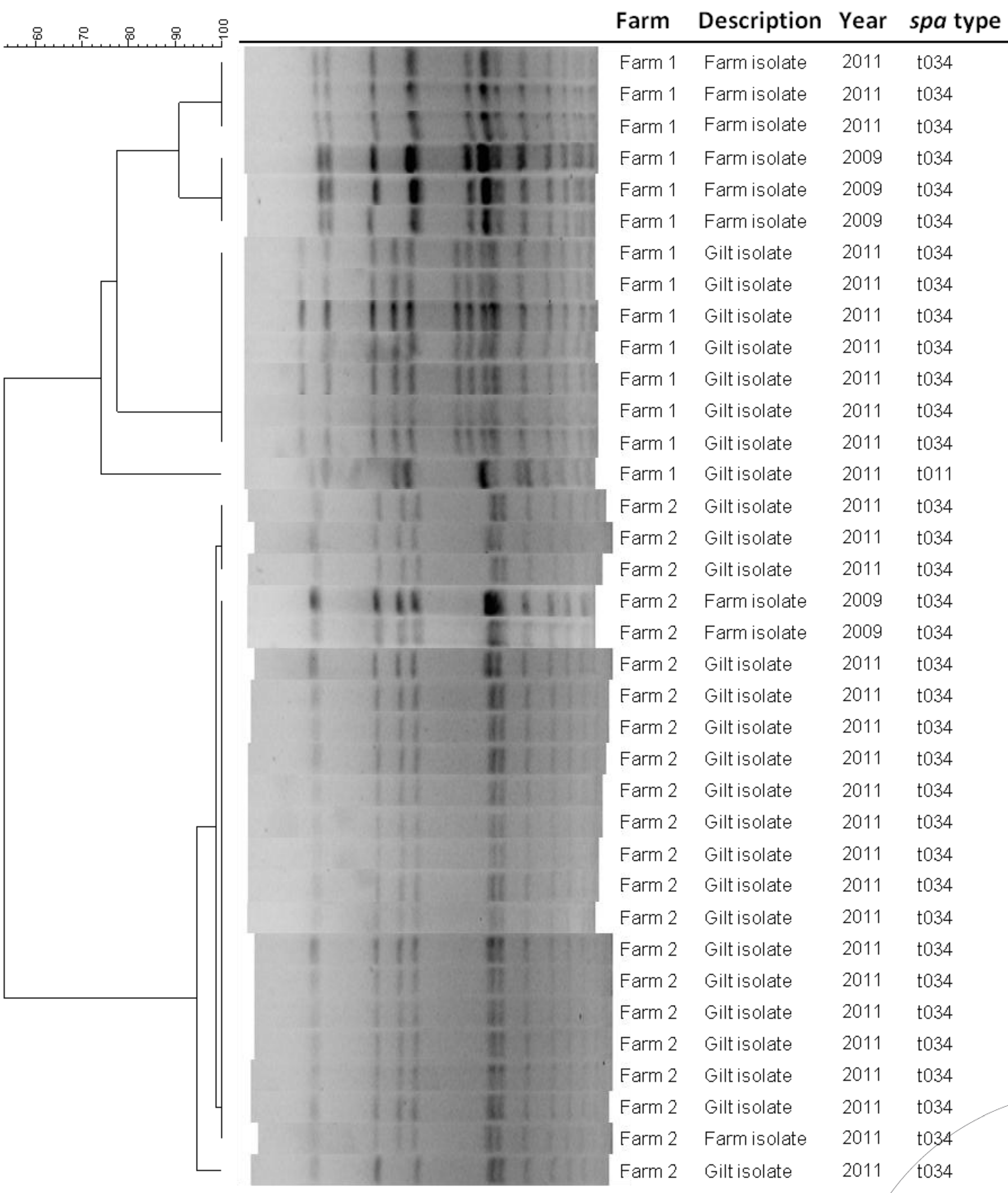

Figure 2. Genetic diversity of 36 MRSA CC398 isolates from 2 Danish pig farms and purchased gilts sampled before entering the farm. Farm isolates were isolated in this study and in another study conducted at the same farms in 2009.


Grundmann, H., Huijsdens, X.W., 2010. PFGE diversity within the methicillin-resistant Staphylococcus aureus clonal lineage ST398. BMC Microbiol. 9, 10, Broens, E.M., Graat E.A., van der Wolf, P.J., van de Giessen, A.W., van Duijkeren, E. Wagenaar, J.A., van Nes, A. Mevius, D.J., de Jong, M.C., 2010. MRSA CC398 in the pi Harmsen, D., Claus, H., Witte, W., Rothganger, J., Claus, H., Turnwald, D., Vogel, U., 2003 Typing of methicilin-resistant Staphylococcus aureus in a university hospital setting by Microbiol. 41, 5442-5448. 